\title{
Magnetic field dependence of triplet-singlet relaxation in quantum dots with spin-orbit coupling
}

\author{
Juan I. Climente, ${ }^{1, *}$ Andrea Bertoni, ${ }^{1}$ Guido Goldoni, ${ }^{1,2}$ Massimo Rontani, ${ }^{1}$ and Elisa Molinari ${ }^{1,2}$ \\ ${ }^{1}$ CNR-INFM S3, Via Campi 213/A, 41100 Modena, Italy \\ ${ }^{2}$ Dipartimento di Fisica, Università degli Studi di Modena e Reggio Emilia, Via Campi 213/A, 41100 Modena, Italy
}

(Received 8 September 2006; revised manuscript received 7 December 2006; published 12 February 2007)

\begin{abstract}
We estimate the triplet-singlet relaxation time due to spin-orbit coupling assisted by phonon emission in weakly confined quantum dots. Calculations are performed taking into account Coulomb and spin-orbit interactions exactly within the full configuration interaction method, and Fermi golden rule. Our results for two and four electrons show that different triplet-singlet relaxation trends observed in recent experiments under magnetic fields can be understood within a unified theoretical description, as the result of the competition between spin-orbit coupling and phonon emission efficiency. Moreover, we show that properly designed QD structures may give access to very long-lived triplet states as well as to selective population of the triplet Zeeman sublevels.
\end{abstract}

DOI: 10.1103/PhysRevB.75.081303

PACS number(s): 73.21.La, 71.70.Ej, 72.10.Di, 73.22.Lp

Semiconductor quantum dots (QDs) are called to play a central role in the emerging field of spintronics, because their zero-dimensional confinement constitutes an optimal environment to manipulate the spin of bound electrons. ${ }^{1}$ Understanding the spin relaxation in these structures is of utmost interest for their eventual use in practical devices. This has triggered a large number of experimental works in the last few years, where two main classes of spin transitions have been investigated, namely the spin-flip between singleelectron Zeeman sublevels ${ }^{2}$ and the triplet-singlet (TS) transition in QDs with an even number of electrons. ${ }^{3-6}$ Remarkably, while the former systems have received much theoretical attention, ${ }^{2,7}$ the understanding of the latter is still rather limited. In particular, the TS relaxation due to spinorbit (SO) coupling-which is often the dominant spin relaxation mechanism in semiconductor QDs-has only briefly been addressed in two-electron QDs, ${ }^{8,9}$ and many relevant features observed in experiments remain uncomprehended.

One such case is the role of an external magnetic field: experimental measurements away from the TS anticrossings suggest that the influence of axial fields on the spin relaxation is fairly weak, ${ }^{3,4}$ in strong contrast with the singleelectron case. ${ }^{2}$ Besides, in the vicinity of the TS anticrossing, both increased ${ }^{4}$ and decreased ${ }^{5}$ relaxation times have been reported. In this context, a coherent picture describing the effect of an axial magnetic field on the TS spin relaxation time is on demand.

In this work, we study the TS spin relaxation due to SO coupling in circular QDs with weak lateral confinement. Acoustic phonon emission, assisted by SO interaction, has been shown to be the dominant relaxation mechanism in this kind of QDs when cotunneling and nuclei-mediated relaxation are reduced. ${ }^{2,4}$ We show that the current experimental evidence $^{3-5}$ can be reconciled within a unified picture, where the field dependence of the relaxation time is determined by the interplay between SO coupling and phonon emission efficiency. Furthermore, we show that such interplay can be tailored in order to obtain improved spin lifetimes.

In weakly confined QDs, correlation effects may strongly influence charge and spin excitations. This can be particularly important in the four-electron system, where exchange and correlation terms may be comparable to quantum con- finement energies. ${ }^{10,20}$ Our method of choice is then the full configuration interaction (FCI). ${ }^{11}$ The single-electron states are calculated within the effective mass approximation for a typical GaAs/AlGaAs QD, with confinement potential $V(\mathbf{r})=V_{z}(z)+1 / 2 m^{*} \omega_{0}^{2}\left(x^{2}+y^{2}\right), V_{z}(z)$ representing the (finite) vertical confinement of a quantum well of thickness $W, \hbar \omega_{0}$ being the single-electron energy spacing of a lateral twodimensional harmonic trap, and $m^{*}$ the effective mass. The lateral confinement is much weaker than the vertical one, and a magnetic field, $B$, is applied along $z$. Under these conditions, the low-lying single-electron states are well described by the product of Fock-Darwin spectrum and the lowest eigenstate of the quantum well. ${ }^{12}$ The few-electron states are then written as $\left|\Psi_{i}\right\rangle=\sum_{j} c_{j i}\left|\Phi_{j}\right\rangle$, where the Slater determinants, $\left|\Phi_{j}\right\rangle=\Pi_{\gamma_{j}} c_{\gamma_{j}}^{\dagger}|0\rangle$, are obtained by filling in the singleelectron spin-orbitals $\gamma$ with $N$ electrons in all possible ways $\left(c_{\gamma}^{\dagger}|0\rangle\right.$ creates an electron in level $\left.\gamma\right)$. The fully interacting Hamiltonian is numerically diagonalized, exploiting orbital and spin symmetries. ${ }^{11}$ The single-electron levels $\gamma$ can be classified by their radial quantum number $n=0,1 \ldots$, azimuthal angular momentum $m=0, \pm 1 \ldots$, and spin $s_{z}=\uparrow, \downarrow$. In turn, the few-electron states can be labeled by the total azimuthal angular momentum $M$, total spin $S$, its $z$-projection $S_{z}$, and by the number $N=0,1, \ldots$ indexing the energy order.

We introduce the SO coupling via the linear Rashba (SIA) and Dresselhaus (BIA) terms, $\mathcal{H}^{\text {SIA }}$ and $\mathcal{H}^{\text {BIA }}$, respectively. ${ }^{13}$ For a quantum well grown along the [001] direction, these terms can be written as ${ }^{14} \mathcal{H}^{\mathrm{SIA}}=\frac{\alpha}{i \hbar}\left(\pi^{+} s_{z}^{-}-\pi^{-} s_{z}^{+}\right)$and $\mathcal{H}^{\mathrm{BIA}}=\frac{\beta}{\hbar}\left(\pi^{+} s_{z}^{+}+\pi^{-} s_{z}^{-}\right)$, where $\alpha$ and $\beta$ are the SIA and BIA interaction coefficients for the sample under study. $\pi^{ \pm}$and $s_{z}^{ \pm}$ are ladder operators, which change $m$ and $s_{z}$ by one unit, respectively. Since the few-electron $M$ and $S_{z}$ quantum numbers are given by the algebraic sum of their single-electron counterparts, SIA mixes $\left(M, S_{z}\right)$ states with $\left(M \pm 1, S_{z} \mp 1\right)$ ones, and BIA mixes $\left(M, S_{z}\right)$ states with $\left(M \pm 1, S_{z} \pm 1\right)$ ones.

We diagonalize the SO terms on a basis of few-electron correlated states. ${ }^{15}$ In the general case the SIA and BIA terms break $S, S_{z}$, and $M$ symmetries. However, for GaAs QDs, SO coupling is but a small perturbation and the quantum numbers $S, S_{z}$, and $M$ are approximately conserved except in the vicinity of the anticrossing regions. ${ }^{8,16}$ Thus, we 

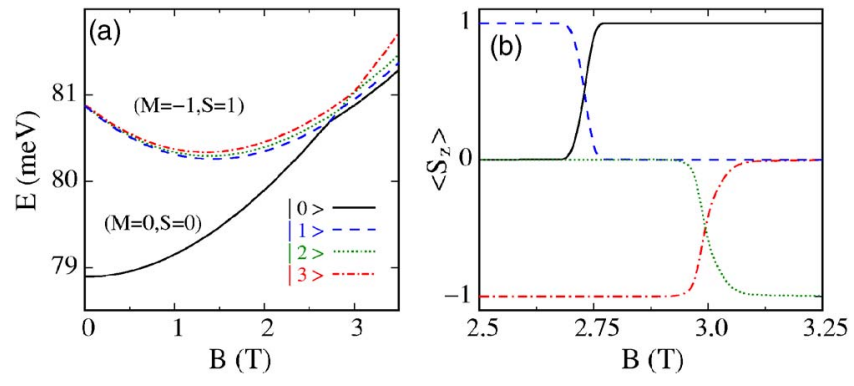

FIG. 1. (Color online) (a) Energy of the four lowest-lying states $|N\rangle$ of a two-electron QD with $W=10 \mathrm{~nm}$ and $\hbar \omega_{0}=4 \mathrm{meV}$ as a function of the magnetic field. (b) $S_{z}$ expectation value of the four lowest energy levels in the region of the singlet-triplet anticrossing.

will still use them for clarity of the discussion. We estimate the relaxation rate at zero temperature due to acoustic phonon emission using Fermi golden rule:

$$
\tau_{b \rightarrow a}^{-1}=\frac{2 \pi}{\hbar} \sum_{\nu \mathbf{q}}\left|\sum_{i j} c_{b i}^{*} c_{a j}\left\langle\Psi_{i}\left|V_{\nu \mathbf{q}}\right| \Psi_{j}\right\rangle\right|^{2} \delta\left(E_{b}-E_{a}-\hbar \omega_{q}\right),
$$

where the SO coupled few-electron states have been written as linear combinations of correlated states $\left|\Psi_{i}\right\rangle . V_{\nu \mathbf{q}}$ is the interaction operator of an electron with a phonon of momentum $\mathbf{q}$ via deformation potential $(\nu=\mathrm{DP})$ or piezoelectric field $(\nu=\mathrm{PZ})$ interaction. $E_{b}, E_{a}$, and $\hbar \omega_{q}$ stand for the energy of the initial and final electron state and of the phonon, respectively. ${ }^{17}$ Note that piezoelectric field scattering, which was neglected in related works, ${ }^{8}$ is actually dominant when the phonon energy is small, ${ }^{7,17}$ so that it provides the main contribution to the relaxation in the interesting regions of TS anticrossings. GaAs material parameters are used in the calculations, ${ }^{17}$ along with a Landé factor $g=-0.44$.

We start our discussion with the two-electron case (see Fig. 1). We use a typical value of the BIA coefficient for a GaAs QD, $\beta=25 \mathrm{meV} \AA$, and a SIA coefficient $\alpha$ $=5 \mathrm{meV} \AA$, as induced by a small asymmetry of the quantum well. The low-lying singlet state and the excited triplet state with three Zeeman sublevels are shown in Fig. 1(a). With increasing magnetic field, the singlet anticrosses with all triplet sublevels. The anticrossing energy gap is very small $(\sim \mu \mathrm{eV})$, as expected for GaAs QDs, ${ }^{3}$ and it is particularly small for the $S_{z}=0$ triplet sublevel. This is because the BIA (SIA) mixes the singlet with the triplet $S_{z}=-1(+1)$ sublevel, but does not mix states with $\Delta S_{z}=0$, which, therefore, takes place only indirectly through higher-lying states. Figure 1(b) illustrates the expectation value $\left\langle S_{z}\right\rangle$ of the four lowest-lying levels around the TS anticrossing. One can see that SO interaction barely affects the spin quantum numbers except in a narrow magnetic field range around the anticrossings. ${ }^{8}$

In Fig. 2 we analyze the relaxation time from the three lowest excited to the ground state of the two-electron QD. The left-hand (right-hand) panel corresponds to structures without (with) SIA. It is found that the relaxation time first decreases slowly with the magnetic field, and then it suddenly increases in the anticrossing region $(B$ $\sim 2.25-3.25 \mathrm{~T}){ }^{18}$ Note that the rather weak dependence with the field before the anticrossing agrees well with TS relaxation measurements in Refs. 3 and 4, the decreased relaxation time before the anticrossing has been reported in Ref. 5, and the peak in the anticrossing region can be associated with the long triplet lifetimes for eight-electron QDs with small TS energy splittings. ${ }^{4}$

The general trends described above can be explained by the opposite effect of the magnetic field on the SO mixing and the phonon emission efficiency. On the one hand, as the TS energy splitting decreases, SO interaction couples the states more efficiently, favoring spin relaxation. On the other hand, the phonon energy decreases, reducing the efficiency of the electron-phonon interaction. The latter effect, which follows from the different orbital quantum numbers of the initial and final electron states, occurs at a rate that is determined by the ratio between the acoustic phonon wavelength and the dimensions of the QD. ${ }^{7,17,19}$ For usual weakly confined QDs, the effect of the magnetic field on the SO interaction and phonon emission turns out to be mostly of similar magnitude, which explains the weak changes of the relaxation time. At the anticrossing point, in spite of the fact that SO mixing becomes very important [see Fig. 1(b)], the phonon energy is so small (few $\mu \mathrm{eV}$ ) that the spin relaxation is strongly suppressed. It is worth pointing out that this result, consistent with recent experiments, ${ }^{4,22}$ is opposed to previous claims in the literature, where maximum relaxation rate was predicted at the anticrossing point. ${ }^{8}$

As stated before, the dimensions of the QD are known to play an important role in determining the phonon emission efficiency. ${ }^{7,17,19}$ One could specifically design QDs whose

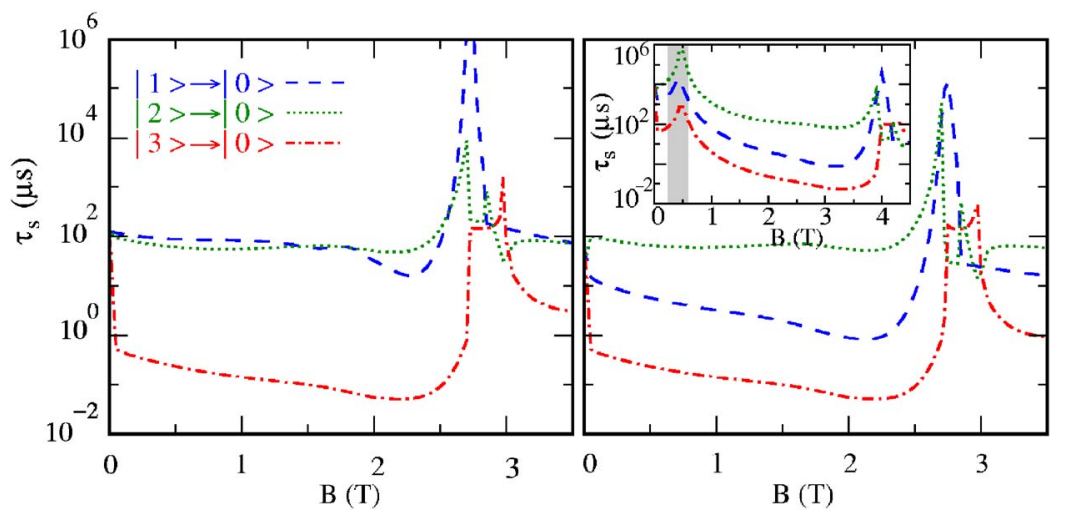

081303-2
FIG. 2. (Color online) Spin relaxation time of the three lowest excited states in a two-electron QD vs magnetic field. $W=10 \mathrm{~nm}, \hbar \omega_{0}=4 \mathrm{meV}$. The left-hand (right-hand) panel shows the case without (with) SIA. Note that before the anticrossing peak, $|N\rangle=1,2,3$ correspond to the triplet sublevels $S_{z}=+1,0,-1$, respectively. The inset shows the relaxation time for a QD with $W=14 \mathrm{~nm}$ and $\hbar \omega_{0}=5 \mathrm{meV}$, the shaded area highlighting the magnetic field window of geometrically reduced phonon emission (see text). 

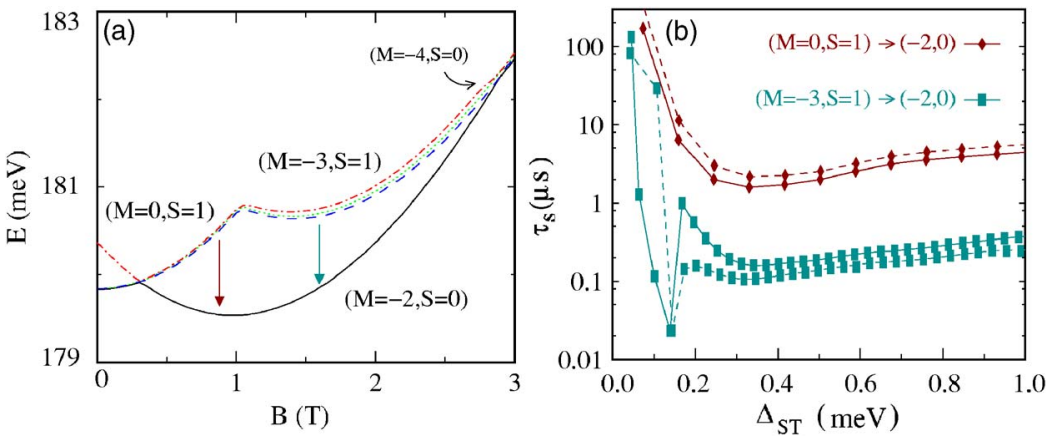

FIG. 3. (Color online) (a) Energy of the four lowest-lying levels of a four-electron QD as a function of the magnetic field. The QD has $W=10 \mathrm{~nm}$ and $\hbar \omega_{0}=4 \mathrm{meV}, \alpha=15 \mathrm{meV} \AA$, and $\beta=25 \mathrm{meV} \AA$. Arrows are used to indicate the two different spin transitions we compare. (b) Average triplet lifetime for the two spin transitions, as a function of the singlet-triplet energy splitting. $\beta=25 \mathrm{meV} \AA$, and solid (dashed) lines are used for $\alpha=0(\alpha=15) \mathrm{meV} \AA$. geometry minimizes electron-phonon coupling. ${ }^{9,17}$ In such a case, phonon efficiency is no longer balanced with the SO mixing effect, and then a very different magnetic field dependence of $\tau_{s}$ as compared to the usual case may be found. An example is illustrated in the inset of Fig. 2, where the relaxation time changes by orders of magnitude with $B$ before reaching the TS anticrossing $(B \sim 4 \mathrm{~T})$, in contrast to the weak field dependence of the general case.

An interesting feature in Fig. 2 inset is the peak at $B \sim 0.5 \mathrm{~T}$ (shaded area), which comes from the geometrically induced suppression of the phonon emission occurring when the quantum well width is a multiple of the phonon wavelength $z$ projection. ${ }^{17}$ This feature may give access to very long-lived triplet states at finite values of the magnetic field, ${ }^{21}$ where phonon-induced relaxation is usually the dominant scattering mechanism. Moreover, the peak takes place at a $B$ value where the initial and final electron states are wellresolved energetically, which renders this phonon bottleneck more useful than the one of the TS anticrossing. The position and depth of this kind of lifetime maxima depend on the QD height and the emitted phonon energy. ${ }^{17}$ Therefore, they are almost independent of the SO interaction in the structure, which in GaAs has a negligible influence on the phonon energy.

Next, we focus on the effect of the separate SIA and BIA contributions over the spin relaxation by comparing the lefthand and right-hand panels of Fig. 2 in the magnetic field region before the TS anticrossing. When only BIA terms are present (left-hand panels), the singlet mixes directly with the higher-lying $\left(S_{z}=-1\right)$ Zeeman sublevel of the triplet. As a result, relaxation from such Zeeman sublevel (dotted-dashed line) is about two orders of magnitude faster than that from the $S_{z}=0,+1$ sublevels, and it exhibits a stronger dependence on the field. When a small SIA is switched on (righthand panels), direct mixing of the singlet with the triplet $S_{z}=+1$ sublevel is enabled. This accelerates the relaxation from this sublevel (dashed line) in one order of magnitude and introduces a stronger dependence on $B$. It is worth noting that the order-of-magnitude damping of the relaxation time due to the SIA is present away from the anticrossing region, where the effect of the SO interaction on $\left\langle S_{z}\right\rangle$ is barely visible [see Fig. 1(b)]. From the above discussion it follows that in a magnetic field both SIA and BIA play an important role in determining the TS spin relaxation time, as opposed to the well-known single-electron case, where the relaxation is mainly due to SIA. ${ }^{2}$ Moreover, we see that the lifetimes of the triplet Zeeman sublevels may strongly differ depending on the relative SIA and BIA contributions. This may be useful to selectively populate the triplet sublevels.

We now investigate the TS spin relaxation in a fourelectron QD. The energy spectrum of the lowest-lying triplet and singlet states in a magnetic field, plotted in Fig. 3(a), is very different from that of the two-electron case, but it closely resembles the one found experimentally for eightelectron QDs in Ref. 4 (except for the absence of eccentricity features in the zero field limit $\left.^{23}\right)$.

Here, we investigate the spin relaxation time in the region $B \sim 0.3-3 \mathrm{~T}$, where the ground state is a singlet $(M=-2)$ and the first excited state is a triplet with two possible values of the angular momentum, depending on the magnetic field: for $B<1 \mathrm{~T}$ the angular momentum is $M=0$, and for $B$ $>1 \mathrm{~T}$ it is $M=-3$. These states are well separated from higher-lying states, so they might be used as a two-level system for quantum computation purposes. We compare the lifetimes of both triplet states in Fig. 3(b), where the averaged relaxation time of the three Zeeman sublevels are plotted as a function of the singlet-triplet energy splitting, $\Delta_{S T}$, with (dashed lines) and without (solid lines) SIA (BIA is present in both cases). The qualitative behavior is similar for both states: the lifetime is roughly constant for $\Delta_{S T}$ $>0.25 \mathrm{meV}$, and it increases for $\Delta_{S T}<0.25 \mathrm{meV}$. This behavior, which is in agreement with the experimental findings of Ref. 4, can be understood in the same terms of compensation between SO coupling and phonon emission efficiency as in the two-electron cases studied above. The strong dip of the $M=-3$ triplet at $\Delta_{S T} \sim 0.15 \mathrm{meV}$ is due to the anticrossing of the upper Zeeman sublevel with the $M=-4$ singlet at strong magnetic fields [at $B \sim 2.8 \mathrm{~T}$ in Fig. 3(a)], which strongly enhances spin relaxation, and it can be related to similar features detected in experiments. ${ }^{4}$ For smaller $\Delta_{S T}$, though, the small phonon energy again leads to increased lifetimes.

An important result shown in Fig. 3(b) is that the average lifetime of the triplets differs by over one order of magnitude depending on their angular momentum, regardless of the (here fairly strong) SIA. This is because the $M=0$ triplet differs from the $M=-2$ singlet ground state in two quanta of angular momentum, and therefore direct $\mathrm{SO}$ mixing is not possible. In contrast, direct mixing is possible for the $M$ $=-3$ triplet, and this makes the relaxation much faster. It then follows that, by using four-electron QDs instead of twoelectron ones, one can use an external magnetic field to select excited states whose spin transition is "forbidden" even in the presence of linear SO interaction. This result is consistent with recent measurements, where different lifetimes 
were observed for triplet states with different orbital quantum numbers. ${ }^{4}$ However, in the experiment the triplet lifetimes changed by a factor of 2 only. The main reason for this difference is probably the ellipticity of their QDs, which mixes states with different angular momenta and hence weakens the efficiency of the $\Delta M= \pm 1$ selection rule.

In summary, we have estimated the TS spin relaxation time due to SO coupling in weakly confined GaAs/AlGaAs QDs. Experimentally observed trends of TS relaxation in magnetic fields ${ }^{3-5}$ are well understood in terms of the competing SO coupling and phonon emission efficiency. Significant differences have been found as compared to the wellknown single-electron spin-flip case. We predict very long triplet lifetimes using QD geometries that lead to suppressed phonon emission. Improved lifetimes can also be obtained in four-electron QDs by selecting triplet states which do not fulfill the $\Delta M= \pm 1$ selection rule.

Finally, we mention that, upon completion of this work, we have learned about parallel experiments which provide continuous measurements of the spin relaxation time vs magnetic field in two-electron QDs, from zero field to the TS anticrossing point. ${ }^{22}$ The behavior observed is in qualitative agreement with that we have predicted in the general case of Fig. 2, which confirms the combined role of phonon scattering and spin-orbit mixing described in this paper. Quantitative discrepancies with the experiment may be ascribed to differences in the QD structural parameters, as well as to the large SO parameters we use, as compared to those inferred in the experiment, which greatly enhance spin-orbit mixing and hence the relaxation rates in our calculations.

The authors are grateful to X. Cartoixa, M. Florescu, and F. Troiani for discussions. The authors acknowledge support from the Italian Ministry for University and Research under FIRB Grant No. RBIN04EY74, CINECA Calcolo parallelo 2006, and Marie Curie IEF Contract No. MEIF-CT-2006023797.
*Electronic address: climente@unimore.it; URL: www.nanoscience.unimore.it

${ }^{1}$ I. Zutic, J. Fabian, and S. Das Sarma, Rev. Mod. Phys. 76, 323 (2004); M. Atatüre et al., Science 312, 551 (2006).

${ }^{2}$ For a review see, e.g., W. A. Coish et al., Phys. Status Solidi B 243, 3658 (2006).

${ }^{3}$ T. Fujisawa et al., Nature (London) 419, 278 (2002).

${ }^{4}$ S. Sasaki, T. Fujisawa, T. Hayashi, and Y. Hirayama, Phys. Rev. Lett. 95, 056803 (2005).

${ }^{5}$ R. Hanson, L. H. Willems van Beveren, I. T. Vink, J. M. Elzerman, W. J. M. Naber, F. H. L. Koppens, L. P. Kouwenhoven, and L. M. K. Vandersypen, Phys. Rev. Lett. 94, 196802 (2005).

${ }^{6}$ J. R. Petta et al., Science 309, 2180 (2005).

${ }^{7}$ P. Stano and J. Fabian, Phys. Rev. B 74, 045320 (2006).

${ }^{8}$ M. Florescu et al., Physica E (Amsterdam) 22, 414 (2004); M. Florescu and P. Hawrylak, Phys. Rev. B 73, 045304 (2006).

${ }^{9}$ D. Chaney and P. A. Maksym, Phys. Rev. B 75, 035323 (2007).

${ }^{10}$ C. P. Garcia, V. Pellegrini, A. Pinczuk, M. Rontani, G. Goldoni, E. Molinari, B. S. Dennis, L. N. Pfeiffer, and K. W. West, Phys. Rev. Lett. 95, 266806 (2005).

${ }^{11}$ M. Rontani et al., J. Chem. Phys. 124, 124102 (2006).

${ }^{12}$ S. M. Reimann and M. Manninen, Rev. Mod. Phys. 74, 1283 (2002).

${ }^{13}$ SIA and BIA stand for structure and bulk inversion asymmetry, respectively. Note that, while BIA is always present in zincblende QDs, SIA is present only if the potential in the $z$ direction is asymmetric.

${ }^{14}$ Y. A. Bychkov and E. I. Rashba, J. Phys. C 17, 6039 (1984); G. Dresselhaus, Phys. Rev. 100, 580 (1955); X. Cartoixa, L. W.
Wang, D. Z.-Y. Ting, and Y. C. Chang, Phys. Rev. B 73, 205341 (2006).

${ }^{15}$ Well-converged few-electron states are obtained with a basis of 56 single-electron spin-orbitals, which for four electrons may form up to $\sim 3 \times 10^{5}$ Slater determinants. The SO interaction Hamiltonian is diagonalized in a basis of 42 fully correlated few-electron states, which leads to convergence in the spin relaxation rate within $2 \%$.

${ }^{16}$ P. Pietiläinen and T. Chakraborty, Phys. Rev. B 73, 155315 (2006).

${ }^{17}$ J. I. Climente, A. Bertoni, G. Goldoni, and E. Molinari, Phys. Rev. B 74, 035313 (2006); J. I. Climente, A. Bertoni, M. Rontani, G. Goldoni, and E. Molinari, ibid. 74, 125303 (2006).

${ }^{18}$ The sharp variation in the relaxation time of Fig. 2 as the magnetic field is switched on is due to removal of the level order imposed by SO interaction at zero field (Ref. 16). However, in GaAs QDs SO interaction is energetically small and this level order will already be removed at $B=0$ by any small deviation from the perfect circular confinement. Thus, we do not expect it to be observable and do not discuss it here.

${ }^{19}$ U. Bockelmann, Phys. Rev. B 50, 17271 (1994).

${ }^{20}$ A. Bertoni, M. Rontani, G. Goldoni, and M. Molinari, Phys. Rev. Lett. 95, 066806 (2005).

${ }^{21}$ A similar feature, concerning phonon scattering suppression with regard to spin relaxation, has been recently put forward by other authors in Ref. 9. Spin-relaxation suppression may be also achieved by quenching the spin-orbit mixing as shown in C. F. Destefani and S. E. Ulloa, Phys. Rev. B 72, 115326 (2005).

${ }^{22} \mathrm{~T}$. Meunier et al., cond-mat/0609726 (to be published).

${ }^{23}$ Y. Tokura et al., Physica B 298, 260 (2001). 